\title{
Observer-based tracking control for single machine infinite bus system via flatness theory
}

\author{
Mohammad Pourmahmood Aghababa ${ }^{1}$, Bogdan Marinescu $^{2}$, Florent Xavier $^{3}$ \\ ${ }^{1,2}$ Ecole Centrale de Nantes, LS2N, France \\ ${ }^{3}$ Réseau de Transport d'Electricité (RTE), France
}

\begin{abstract}
Article Info
Article history:

Received Dec 11, 2019

Revised Jul 31, 2020

Accepted Aug 17, 2020

\section{Keywords:}

Critical clearing time

Flat system

Input constraints

State observer

Transient stability

ABSTRACT

In this research, we aim to use the flatness control theory to develop a useful control scheme for a single machine connected to an infinite bus (SMIB) system taking into account input magnitude and rate saturation constraints. We adopt a fourth-order nonlinear SMIB model along an exciter and a turbine governor as actuators. According to the flatness-based control strategy, first we show that the adopted nominal SMIB model is a flat system. Then, we develop a full linearizing state feedback as well as an outer integral-type loop to ensure suitable tracking performances for the power and voltage as well as the angular velocity outputs. We assume that only the angular velocity of the generator is available to be measured. So, we provide a linear Luenberger observer to estimate the remaining states of the system. Also, the saturation nonlinearities are transferred to the linear part of the system and they are canceled out using their estimations. The efficiency and usefulness of the proposed observer-controller against faults are illustrated using simulation tests in Eurostag and Matlab. The results show that the clearing critical time of the introduced methodology is larger than the classical control approaches and the proposed observer-based flatness controller exhibits over much less control energy compared to the classic IEEE controllers.
\end{abstract}

This is an open access article under the CC BY-SA license.

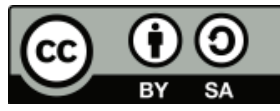

\section{Corresponding Author:}

Bogdan Marinescu

Ecole Centrale de Nantes

LS2N, Nantes, France

Email: Bogdan.Marinescu@ec-nantes.fr

\section{INTRODUCTION}

Nowadays, the electricity has become as an important and vital component of the life and industry. So, the electrical power networks should be in a secure operation with a reasonable stability margin to produce the demanded electricity. The ability of a power system in maintaining in the machines synchronous operation point after occurrence of a disturbance and/or fault is usually interpreted as its transient stability concept. To retain the power system stability in a suitable limit in the event of uncertainties, faults and disturbances, it is necessary to add control actions, such as exciters and governors, to the system to improve dynamics till the circuit breaker opening and reclosing times [1]. The critical clearing time (CCT) is one useful and applied factor to measure the transient stability margin of a power machines. The CCT stands for the maximum time during which a fault can be applied without missing the system's stability. Such a stability margin depends on the design of the controls of generators connected to the grid [2].

To enhance the CCT of a grid, some control devices should be designed and implemented in the network. To synthesis and analyze the performance of the controllers on the CCT of a power grid, a single 
machine connected to an infinite bus (SMIB) power system model is usually adopted to avoid the unnecessary complexity of the power system in the control design phase. Generally speaking, there are two main controller classes for this case: (i) standard controllers and (ii) advanced control techniques. The first class belongs to the well-known standard IEEE controllers. More details about the classic IEEE controllers can be found in [3, 4]. Although the classic IEEE controllers are simple, their parameters are needed to be appropriately adjusted and their stability regions are limited. In the works [5-7] some intelligent heuristic optimization methods have been proposed for finding the suitable parameters of the regulators. However, since their approaches requires implementation of some iterative numerical algorithms, their practical implementations will be difficult in online and fast response needed situations. On the other hand, the approaches in the second class use some so-called advanced control strategies to enhance the transient stability of the power machines. There are several examples in the literature for this category which include sliding mode control [8], fuzzy control [9], nonlinear control [10], dynamic inversion control [11] and optimal control [12], etc.

In [13], a power system stabilizer has been proposed for synchronous machines based on conventional fuzzy-PID and type-1 fuzzy controller combined with a sliding mode control strategy. The work [14] has proposed an adaptive wavelet network-based nonlinear excitation control for power systems without considering the governor dynamics. To improve the stability of the voltage regulation and to enhance the damping of low frequency power system oscillations of SMIB systems, an extended reduced-order observer along with an automatic voltage regulator has been developed in [15]. In [16], the relationship between transient stability/ instability and concavity/convexity of the phase-plane trajectory has been found and a transient instability criterion has been derived for real-time instability detection and the SMIB system has been stabilized. Rout et al [17] have showed that the SMIB system can possess chaotic and oscillatory dynamics when the system parameters fall into a certain area. Accordingly, they have designed an adaptive controller based on LaSalle's invariant principle to make the system oscillations damped. The paper [18] has investigated the problem of transient stability and voltage regulation for a SMIB system via a modified backstepping control design method. However, most of the previous works either have not considered the effects of input saturations, they have been designed for some simplified linear and/or nonlinear models of the SMIB, there are usually steady state errors on the outputs of their methods or they have assumed that all the states of the system are available to be measured.

The concept of differentially flat nonlinear systems was first introduced by Fliess et al [19, 20]. The scheme is an extension from the input-output scheme with zero internal dynamics. A system is considered to be differentially flat if all its state variables and its control inputs can be expressed as functions of one single algebraic variable which is the so-called flat output, and also as functions of the flat-output's derivatives. The differential flatness property enables the transformation of the nonlinear system's dynamics into the linear canonical form and the design of a state feedback controller through the application of pole placement techniques in the linearized equivalent model of the system. The construction of the feedback law is done by a simple inversion of system equations with respect to the system input. Although this technique has been applied to several nonlinear and linear mechanical systems [21-23], its application to the control of power systems has been limited to a few works [24-26] and [27]. However, the previous works have not focused on the transient stability margin and they either have not carried out the effects of the actuator saturations or they have assumed that all the states of the synchronous machines are available to be measured.

In this research, inspired by the flatness control theory, we propose a full linearizing state feedback for the system to cancel out the nonlinearities of the system and to obtain a linear canonical (Brunovsky) form for it. Then, we transfer the input saturation nonlinearities of the system to the adopted linear part of the model. To make the obtained linear system to be controllable and observable, some modifications are done on the nonlinear feedback control to modify the linear matrix of the system. Subsequently, a full order state linear observer is designed for the system to estimate the states of the model using the only available output, i.e. the angular velocity. To provide tracking of voltage and power reference commands, integral outer loops are added to the linear part of the system. The Kalman filter and the linear quadratic regulator approaches are adopted to derive the linear observer and controller, respectively, and to construct the framework of the final augmented system. Finally, the efficiency and applicability of the proposed observer-based state feedback controller are revealed using some illustrative examples simulated in the presence of short-circuit faults and magnitude and rate saturations of input signals, where the superiority of the introduced controller is revealed for its robustness against unwanted faults increasing CCT of the machines and its low implementation costs reducing the control energy required for the stabilization of the system compared to the classic IEEE control schemes. 


\section{SYSTEM MODELING AND PROBLEM FORMULATION}

Figure 1 shows a simple representation of a power system consisting of a single machine connected to an infinite bus through a transmission line with impedance $Z=r_{e}+j x_{e p}$. In the sequel, we will present the one-axis model, named also flux decay model, of the SMIB power system where the reader may refer to [1] for more details.

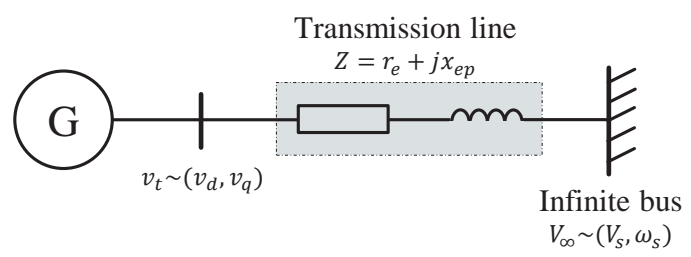

Figure 1. A SMIB power system

\subsection{One-axis model of a SMIB}

The mechanical dynamics of the generator, which correspond to the rotor's relative angle $(\delta)$ and the angular velocity $(\omega)$, are described by:

$$
\begin{aligned}
\dot{\delta} & =\left(\omega-\omega_{s}\right) \omega_{0} \\
\dot{\omega} & =\frac{\omega_{s}}{2 H}\left(T_{m}-T_{f w}-T_{e}\right)
\end{aligned}
$$

where $\omega_{s}$ is the rated synchronous speed in p.u, $\omega_{0}$ is the rated synchronous speed in $\mathrm{rad} / \mathrm{s}, H$ is the machine's inertia, $T_{m}$ represents the mechanical torque applied to the shaft, $T_{f w}=D\left(\omega-\omega_{s}\right)$ is a friction windage torque with a damping coefficient $D$ and $T_{e}$ is the electrical torque defined by the following expression

$$
T_{e}=i_{q} e_{q}^{\prime}+\left(x_{q}-x_{d}^{\prime}\right) i_{d} i_{q}
$$

where $x_{q}$ is the synchrounous reactance of the generator in quadratic axis and $x_{d}^{\prime}$ is its transient reactance in direct axis.

The mechanical power, denoted $P_{m}$, is defined as:

$$
P_{m}=T_{m} \omega
$$

Inserting in 2) $T_{e}$ given by (3) and $T_{m}$ given by (4) and assuming $D=0$, one gets

$$
\dot{\omega}=\frac{1}{2 H}\left(T_{m}-i_{q} e_{q}^{\prime}-\left(x_{q}-x_{d}^{\prime}\right) i_{d} i_{q}\right)
$$

The dynamic of the induced voltage on the $q$-axis, denoted $e_{q}^{\prime}$, is given by:

$$
\dot{e_{q}^{\prime}}=\frac{1}{T_{d 0}^{\prime}}\left(-e_{q}^{\prime}-\left(x_{d}-x_{d}^{\prime}\right) i_{d}+E_{f d}\right)
$$

where $E_{f d}$ represents the voltage across the rotor field coil, which is considered as an input for the SMIB model to be designed.

The mathematical equation of the governor dynamics is given below.

$$
\dot{P}_{s v}=\frac{1}{T_{g}}\left(T_{c c}-P_{s v}+K_{g}\left(\omega-\omega_{s}\right)\right)
$$

in which $T_{c c}$ is a constant to be designed as a reference for mechanical torque and $P_{s v}$ is the steam valve position and it represents the second input for the SMIB model.

The turbine dynamical equation is also given by:

$$
\dot{T}_{m}=\frac{1}{T_{c h}}\left(P_{s v}-T_{m}\right)
$$


The currents in $d q$-coordinates $\left(i_{d}, i_{q}\right)$ are obtained by solving a set of algebraic equations, which represents the interaction of the machine with the power grid:

$$
\left\{\begin{array}{l}
i_{d}=c_{1}+c_{2} e_{q}^{\prime} \\
i_{q}=c_{3}+c_{4} e_{q}^{\prime}
\end{array}\right.
$$

with

$$
\begin{aligned}
& c_{1}=-\frac{\left(r_{s}+r_{e}\right) \sin (\delta)+\left(x_{q}-x_{e p}\right) \cos (\delta)}{\left(r_{s}+r_{e}\right)^{2}+\left(x_{d}^{\prime}+x_{e p}\right)\left(x_{q}+x_{e p}\right)} V_{s} \\
& c_{2}=\frac{x_{q}+x_{e p}}{\left(r_{s}+r_{e}\right)^{2}+\left(x_{d}^{\prime}+x_{e p}\right)\left(x_{q}+x_{e p}\right)} \\
& c_{3}=\frac{\left(x_{d}^{\prime}+x_{e p}\right) \sin (\delta)-\left(r_{s}+r_{e}\right) \cos (\delta)}{\left(r_{s}+r_{e}\right)^{2}+\left(x_{d}^{\prime}+x_{e p}\right)\left(x_{q}+x_{e p}\right)} V_{s} \\
& c_{4}=\frac{r_{e}+r_{s}}{\left(r_{s}+r_{e}\right)^{2}+\left(x_{d}^{\prime}+x_{e p}\right)\left(x_{q}+x_{e p}\right)}
\end{aligned}
$$

Assuming $r_{s}=r_{e}=0$ (i.e., overhead lines with neglectable resistances compared to reactances), the currents $i_{d}, i_{q}$ are simplified as follows:

$$
\begin{aligned}
& i_{q}=\frac{V_{s}}{x_{e p}+x_{d}} \sin (\delta) \\
& i_{d}=\frac{1}{x_{e p}+x_{d}^{\prime}} e_{q}^{\prime}-\frac{V_{s}}{x_{e p}+x_{d}^{\prime}} \cos (\delta)
\end{aligned}
$$

The above dynamics (1)-(10) can be regrouped in the following fifth-order state form:

$$
\dot{x}(t)=f(x(t), u(t)), x\left(t_{0}\right)=x_{0}
$$

where $x_{1}=\delta, x_{2}=\omega, x_{3}=e_{q}^{\prime}, x_{4}=T_{m}, u_{1}=E_{f d}$ and $u_{2}=P_{s v}$.

It is noted that in this work, we will include the governor dynamics (7) in an outer control loop. constraints:

It should be also noted that due to physical limitations, the control inputs have to verify the following

Magnitude limitation: The exciter may generate positive and negative values where symmetric bounds are generally considered:

$$
\left|u_{1}\right| \leq \bar{u}_{1}
$$

However, the steam valve position is limited between 0 (completely closed) and 1 (completely open):

$$
0 \leq u_{2} \leq \bar{u}_{2}
$$

Rate limitation: Beside the magnitude saturation, the variation of steam valve position has to respect some limitations:

$$
\frac{1}{T_{c}} \leq \dot{u}_{2} \leq \frac{1}{T_{o}}
$$

where $T_{o}\left(T_{c}\right)$ is the necessary time to pass from a completely closed (open) position to a completely open (closed) position.

Remark 1: One can use the first-order derivative approximation $\dot{u} \cong \frac{u(t)-u(t-\epsilon)}{\epsilon}$ (where $\epsilon$ is a small constant) to convert the rate saturation to a magnitude saturation as follows:

$$
\frac{1}{T_{c}} \leq \dot{u}_{2} \leq \frac{1}{T_{o}} \Rightarrow \frac{1}{T_{c}} \leq \frac{u_{2}(t)-u_{2}(t-\epsilon)}{\epsilon} \leq \frac{1}{T_{o}} \Rightarrow \frac{\epsilon}{T_{c}}+u_{2}(t-\epsilon) \leq u_{2}(t) \leq \frac{\epsilon}{T_{o}}+u_{2}(t-\epsilon)
$$



will have

Now, defining $u_{2 M i n}=\frac{\epsilon}{T_{c}}+u_{2}(t-\epsilon)$ and $u_{2 M a x}=\frac{\epsilon}{T_{c}}+u_{2}(t-\epsilon)$ and noting to $0 \leq u_{2} \leq \bar{u}_{2}$, we

$$
u_{2 L} \leq u_{2}(t) \leq u_{2 H}
$$

with $u_{2 L}=\max \left\{0, u_{2 M i n}\right\}$ and $u_{2 H}=\min \left\{\bar{u}_{2}, u_{2 M a x}\right\}$.

\subsection{SMIB Model in faulty mode operation}

The appearance of a temporary short-circuit in the transmission line affects considerably the system's stability. Let us consider the case of two parallel transmission lines with the same impedance $Z^{\prime}=2\left(r_{e}+j x_{e p}\right)$. This allows us to preserve the same impedance of the previous transmission line $Z$. The short-circuit will happen at time $t_{c}$ at the middle of the second transmission line. This faulty mode affects the SMIB model by changing its parameters $\left(r_{e}, x_{e p}\right)$ by $\frac{2}{3}\left(r_{e}, x_{e p}\right)$ and $V_{s}$ by $\frac{1}{3} V_{s}$ as reported in [28].

Notice that in practice the short-circuit may happen at any point of the line. It has been examined here in the middle for the sake of simplicity. For example, if the short-circuit appears at the terminal of the generator, $V_{s}$ will be replaced by zero in the dynamics (1)-(10). In this case, the machine's speed increases, with respect to the dynamic (17), as long as the short-circuit is present and depending on its duration (denoted $\Delta t$ ) the machine may lose synchronism.

$$
\dot{\omega}=\frac{\omega_{s}}{2 H} T_{m}
$$

\subsection{Control objectives}

The main control objective for the synchronous machine is to operate at synchronous speed $\omega_{s}$, maintain a constant modulus of the terminal voltage $V_{\text {ref }}$ and achieve a desired mechanical power $P_{\text {ref }}$ under control inputs constraints. Accordingly, we will try to mimic the standard classic IEEE governor model (7) in the closed-loop system. The modulus of the terminal voltage of the synchronous generator, denoted $v_{t}$, is given by:

$$
v_{t}=\sqrt{v_{d}^{2}+v_{q}^{2}}
$$

where:

$$
\left\{\begin{array}{l}
v_{d}=r_{e} i_{d}-x_{e p} i_{q}+V_{s} \sin \left(x_{1}\right) \\
v_{q}=r_{e} i_{q}+x_{e p} i_{d}+V_{s} \cos \left(x_{1}\right)
\end{array}\right.
$$

\section{OBSERVER-BASED NONLINEAR CONTROLLER DESIGN}

In this section, first we show that the nonlinear model SMIB system is flat. Then, we will compensate the effects of the input saturations. Finally, a linear state observer is designed for the system to built the final flatness-based controller.

\subsection{Checking out flatness of SMIB}

Consider a general case of the nonlinear system (11) with $x(t) \in \mathbb{R}^{n}$ and $u(t) \in \mathbb{R}^{m}$. It is said to be differentially flat if and only if there exists a flat output $z(t) \in \mathbb{R}^{m}$ of the form :

$$
z=h\left(x, u, \dot{u}, \ldots, u^{(r)}\right)
$$

such that

$$
\begin{aligned}
& x=\phi\left(z, \dot{z}, \ldots, z^{(q)}\right) \\
& u=\psi\left(z, \dot{z}, \ldots, z^{(q+1)}\right)
\end{aligned}
$$

The components of the flat output $z$ are differentially independent. These equations yield, that for every given trajectory of the flat output $t \rightarrow z(t)$, the evolution of all other variables of the system $t \rightarrow x(t)$ and $t \rightarrow u(t)$ are also given without integration of the system of differential equations

Proposition 1: The one-axis model of the single machine infinite bus power system given by (11) is differentially flat with the output $z=\left[\begin{array}{ll}x_{1} & x_{4}\end{array}\right]^{T}$. 
Proof: We should prove that the remaining states $x_{2}=\omega$ and $x_{3}=e_{q}^{\prime}$ as well as the control inputs $u_{1}=E_{f d}$ and $u_{2}=P_{s v}$ can be written as a function of the flat outputs and their successive derivatives. First, from (2), one gets

$$
\omega=\frac{\dot{\delta}}{w_{0}}+\omega_{s}
$$

So, $x_{2}=\omega$ is stated by the flat output $\delta$. From (2), one obtains the following relation for $e_{q}^{\prime}$.

$$
e_{q}^{\prime}=\frac{T_{m}-\frac{2 H}{w_{0} w} \ddot{\delta} A A V_{s} i_{q}}{(1+A A) i_{q}}
$$

where $A A=\frac{x_{q}-x_{d}^{\prime}}{x_{e p}+x_{d}^{\prime}}$.

The next step is to express the control inputs as functions of the outputs and their derivatives. To this end, in what follows, we replace the control inputs $u_{1}(t)$ and $u_{2}(t)$ with $\operatorname{Sat}\left(u_{1}\right)$ and $\operatorname{Sat}\left(u_{2}\right)$, respectively to include the corresponding saturation nonlinearities. As a result, using (6) and (23), one can obtain

$$
\operatorname{Sat}\left(u_{1}\right)=T_{d 0} e_{q}^{\prime}+e_{q}^{\prime}+\left(x_{d}-x_{d}^{\prime}\right) i_{d}
$$

where

$\dot{e}_{q}^{\prime}=\frac{\left[\dot{T}_{m}-\frac{2 H}{w_{0} w_{s}} \ddot{\delta}+A A V_{s}\left(i q \cos (\delta)-\sin (\delta) i_{q}\right)\right]\left(i_{q}(1+A A)\right)-F(X)}{\left(i_{q}(1+A A)\right)^{2}}$ with $F(X)=\dot{i q}(1+A A)\left(T_{m}-2 H w_{0} w \dot{\delta}+\right.$ $\left.A A V_{s} \cos (\delta) i_{q}\right)$ and $\dot{i q}=\frac{V \operatorname{scos}(\delta)}{x_{e p}+x q}$.

And, according to the (8) one can easily obtain

$$
\operatorname{Sat}\left(u_{2}\right)=T_{c h} \dot{T}_{m}+T_{m}
$$

So, the proof is completed and the selected variables $z=\left[\begin{array}{ll}x_{1} & x_{4}\end{array}\right]^{T}$ are the flat outputs of the SMIB system.

\subsection{Flatness-based linearizing state feedback}

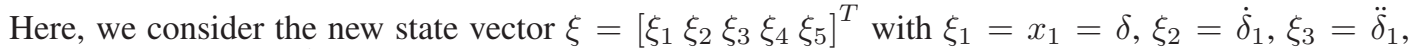
$\xi_{4}=x_{4}=P_{m}$ and $\xi_{5}=\dot{P}_{m}$. In what follows, we conclude a proposition to highlight the equivalence between the nonlinear model of a SMIB power system and a linear controllable one. Before proceeding to the proposition, we first rewrite the saturation function as follows:

$$
\operatorname{Sat}\left(u_{i}\right)=u_{i}+\Delta u_{i}, i=1,2 .
$$

where $\Delta u_{i}$ is defined as follows:

$$
\Delta u_{i}=\left\{\begin{array}{c}
u_{i}^{H}-u_{i}, u_{i} \geq u_{i}^{H} \\
0, u_{i}^{L} \leq u_{i} \leq u_{i}^{H} \\
u_{i}^{L}-u_{i}, u_{i} \leq u_{i}^{L}
\end{array}\right.
$$

Therefore, based on (26) and defining $v_{1}=\ddot{\delta}$ and $v_{2}=\dot{T}_{m}$, the control inputs $u_{1}$ (24) and $u_{2}$ (25) are reformed as (28) with $\Delta v_{1}=-\frac{w_{s} w_{0} \Delta u_{1}}{2 H}$ and $\Delta v_{2}=-\frac{\Delta u_{2}}{T_{c h}}$.

$$
u_{1}=\frac{T_{d 0}\left[\dot{T}_{m}-\frac{2 H}{w_{0} w_{s}}\left(v_{1}+\Delta v_{1}\right)+A A V_{s}\left(i q \cos (\delta)-\sin (\delta) i_{q}\right)\right]\left(i_{q}(1+A A)\right)-T_{d 0} F(X)}{\left(i_{q}(1+A A)\right)^{2}}+\frac{T_{m}-\frac{2 H}{w_{0} w} \ddot{\delta} A A V_{s} i_{q}}{(1+A A) i_{q}}+\left(x_{d}-x_{d}^{\prime}\right) i_{d}
$$

Proposition 2: Under the mapping $\xi=\Phi(x)$ given by:

$$
\Phi(x)=\left(\begin{array}{c}
x_{1} \\
\frac{x_{2}-\omega_{s}}{\omega_{0}} \\
\frac{\left(-x_{3} i_{q}(1+A A)+x_{4}+A A V_{s} i_{q}\right) w_{0} w_{s}}{2 H} \\
x_{4}
\end{array}\right)
$$


and the state feedback given in (28), the nonlinear model of the SMIB is equivalent to the following semi-linear (i.e. linear system with nonlinear uncertainties) controllable system presented in (30).

$$
\dot{\xi}=A \xi+B(v+\Delta v)
$$

where:

$$
v=\left[\begin{array}{l}
v_{1} \\
v_{2}
\end{array}\right], \Delta v=\left[\begin{array}{l}
\Delta v_{1} \\
\Delta v_{2}
\end{array}\right], A=\left[\begin{array}{llll}
0 & 1 & 0 & 0 \\
0 & 0 & 1 & 0 \\
0 & 0 & 0 & 0 \\
0 & 0 & 0 & 0
\end{array}\right], B=\left[\begin{array}{ll}
0 & 0 \\
0 & 0 \\
1 & 0 \\
0 & 1
\end{array}\right]
$$

Proof: By considering the flat variables $x_{1}$ and $x_{5}$, the corresponding Brunovsky form has a full rank controllability matrix, which means that one can propose a state feedback that can linearize the SMIB model (11) $[20,29]$. Using the above diffeomorphism and replacing the control expression (28) in the dynamics of the new coordinates yields the above linear controllable system (30).

Remark 2: It should be noted that although, we have not used the derivatives of the saturation functions of the control inputs in proposition 1 to prove the flatness property of the SMIB system, the available discontinuities in the $\Delta u$ may make the proposed diffeomorphism mapping to be local from the equivalency point of view. However, transferring the discontinuous term $\Delta u$ to the semi-linear side as the term $\Delta v$ and noting to this fact that the discontinuities of $\Delta v$ are exactly equal to those of $\Delta u$ (due to this fact that $\Delta v$ includes the exact $\Delta u$ ), will avoid this problem and, therefore, the proposed diffeomorphism will be global.

Remark 3: It should be noted that although, we have not used the derivatives of the saturation functions of the control inputs in Proposition 1 to prove the flatness property of the SMIB system, the available discontinuities in the $\Delta u$ make the proposed diffeomorphism mapping to be local from the equivalency point of view. In fact, when the control inputs hit the saturation limits, there will not be a one-by-one mapping between the semi-linear Brunovsky system and the corresponding nonlinear SMIB model anymore. However, even in this case, one can design proper linear control inputs $v(t)$ to stabilize the system like an output feedback linearization scheme.

\subsection{Linear state observer design}

From flatness-control point of view, one of the main practical significance of the flat outputs of a dynamical system is that if the flat outputs are measurable, then all the system variables required for feedback can be directly computed without integrating any differential equations. In our case, the rotor's relative angle and the mechanical power are selected as the system flat outputs. However, in practice, these values cannot be easily measured. And, in most practical situations, the angular velocity of the machine is the only available output to be measured. Accordingly, if we want to implement the above-designed controller, we should fist develop an observer for the system to estimate the states of the system using the angular velocity output. In what follows, the observer design procedure for the SMIB system is presented.

Instead of directly developing an observer for the SMIB system given in (1)-(10), we use the equivalent semi-linear system (31) to propose an efficient linear Luenberger observer. To this end, we first select the output matrix of the system as $y=\left[\begin{array}{llll}0 & 1 & 0 & 0\end{array}\right]^{T}$ and check the observability property of the system (31). After checking the rank of the observability matrix, it is revealed that it is singular (it is rank efficient) and, therefore, the system (31) is not observable. To solve this problem, we propose an alternative: modify the linear matrix $A$ in (31) using modifying the linear control inputs $v_{1}$ and $v_{2}$ to obtain an observable system. In this line, we add $\mp T_{m}$ and $\mp \delta$ to the control inputs $u_{1}$ and $u_{2}$ in (28), respectively as formulated in (32).

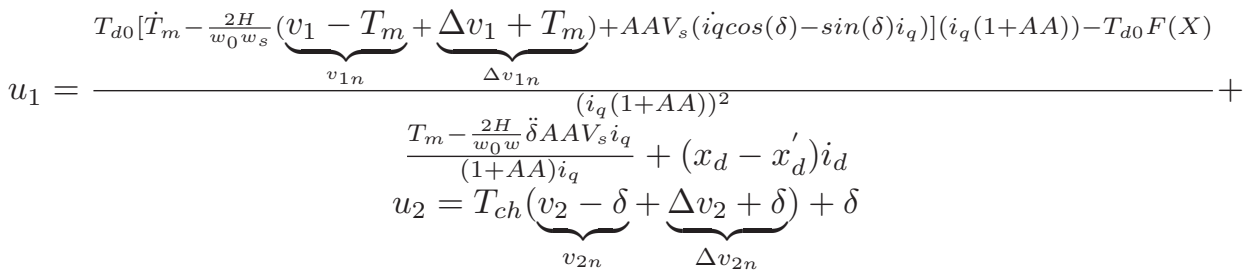


The above modifications are equivalent to put a 1 in the third row-forth column of the matrix $A$ and another 1 in the firth row-fifth column of the matrix $A$ as follows:

$$
A=\left[\begin{array}{llll}
0 & 1 & 0 & 0 \\
0 & 0 & 1 & 0 \\
0 & 0 & 0 & 1 \\
1 & 0 & 0 & 0
\end{array}\right]
$$

Now, the pair $(A, C)$ is observable and the pair $(A, B)$ is controllable (their corresponding observability and controllability matrices are full rank). The linear Luenberger observer is designed using the following dynamics:

$$
\dot{\widehat{\xi}}=A \widehat{\xi}+B V+L(y-C \xi)
$$

where $V=\left[\begin{array}{ll}v_{1 n} & v_{2 n}\end{array}\right]^{T}$ is the linear control input to be designed later.

The observer gain matrix $L$ can be easily obtained using the Kalman command of the Matlab software. So, the estimated states $\widehat{\xi}$ will be used to construct the final controller (including linear controls $v_{1 n}, v_{2 n}$ and nonlinear ones $u_{1}, u_{2}$ ).

Table 1. Typical parameters of a SMIB power system in p.u

\begin{tabular}{lcccccccccccc}
\hline Parameter & $\omega_{s}$ & $V_{s}$ & $T_{d 0}^{\prime}$ & $x_{d}$ & $x_{q}$ & $x_{d}^{\prime}$ & $H$ & $T_{c h}$ & $r_{s}$ & $r_{e p}$ & $D$ & $x_{e p}$ \\
\hline Value & 1 & 1 & 9.6 & 2.38 & 1.21 & 0.336 & 5 & 5 & 0 & 0 & 0 & 0.4 \\
\hline
\end{tabular}

\subsection{Output tracking loop}

The new vector of control $V$ has to guarantee the stability of the SMIB system and achieve the control requirements discussed before. Thus, the state vector $\xi$ has to be driven to a desired state $\xi_{\text {ref }}$, which corresponds to a desired relative angle, a zero relative speed, a zero acceleration and a desired mechanical torque. However, in addition to the driving the states of the semi-linear system to the desired the reference values, for better tracking purposes and good robustness properties, we add an output integrator loop to the system to make it for efficient satisfaction of the control objectives. On the other hand, one needs to cancel out the effects of the semi-linear terms $\Delta v$ in the system. So, the output tracking loop includes three parts: i) an active control for cancelling the semi-linear parts, ii) a feedforward tracking scheme for providing the tracking conditions of linear state space systems, and iii) an integrator loop for including the output errors in the obtained equvalent linear system. These items are explained in the next subsections.

\subsubsection{Active control}

Here, we aim to cancel out the effects of the semi-linear parts $\Delta v_{1 n}$ and $\Delta v_{2 n}$. Noting to the definitions $\Delta v_{1 n}=-\frac{w_{s} w_{0} \Delta u_{1}}{2 H}+T_{m}$ and $\Delta v_{2 n}=-\frac{\Delta u_{2}}{T_{c h}}+\delta$, it is revealed that these terms are known. So, we easily use the so-called active control method [30] to directly remove them by a feedback control. However, it should be noted that we cannot use the states of the nonlinear system; instead, we use the outputs of the derived observer to build estimations for $\Delta v_{1 n}$ and $\Delta v_{2 n}$. Accordingly, the estimated semi-linear parts will be added to the control input $v_{n}$ to cancel out the effects of the corresponding terms. In this line, one can define a new linear control input as follows:

$$
v_{n}=v_{f}-\Delta v
$$

where $v_{f}$ is the new linear control input and $\Delta v=\left[\Delta v_{1 n} \Delta v_{2 n}\right]^{T}$.

Based on the above formulation, the equivalent linear system for the nonlinear model of the SMIB becomes:

$$
\begin{aligned}
& \dot{\xi}=A \xi+B v_{f} \\
& y=C \xi
\end{aligned}
$$




\subsubsection{Feedforward tracking scheme}

Here, we incorporate the reference input into the observer/controller state feedback. This method is useful for tracking of slowly changing references. Consider the state space system (36).

1. We first design a state feedback gain $K$ such that $A-B K$ is stable (with poles at nice locations);

2. Suppose that $\xi_{\text {ref }}$ is the reference input.

We would associate a steady state vector $\xi_{s s}=N_{\xi} \xi_{\text {ref }}$ for any constant reference input $\xi_{\text {ref }}$. Now we define the control to be:

$$
V_{f}=-K\left(\xi-\xi_{s s}\right)+v_{s s}
$$

where $V_{s s}=N_{v_{f}} \xi_{\text {ref }}$ is the steady state control input to maintain $\xi$ at $\xi_{s s}$.

3. To define $N_{v_{f}}$ and $N_{\xi}$, we consider the desired steady state relationships:

$$
\begin{aligned}
& \dot{\xi_{s s}}=A \xi_{s s}+B v_{f s s}=\left(A N_{\xi}+B N_{v_{f}}\right) \xi_{\text {ref }}=0 \\
& y_{s s}=C \xi_{s s}=C N_{\xi} \xi_{\text {ref }}=\xi_{\text {ref }}
\end{aligned}
$$

To make this work for all $\xi_{\text {ref }}$, we need to solve:

$$
\left(\begin{array}{cc}
A & B \\
C & 0
\end{array}\right)\left(\begin{array}{c}
N_{\xi} \\
N_{v_{f}}
\end{array}\right)=\left(\begin{array}{l}
0 \\
1
\end{array}\right)
$$

4. Once $N_{\xi}$ and $N_{v_{f}}$ have been found, we can rewrite the control law to be:

$$
\begin{aligned}
& V_{f}=-K\left(\xi-N_{\xi} \xi_{\text {ref }}\right)+N_{v_{f}} \xi_{\text {ref }} \\
& V_{f}=-K \xi+\bar{N} \xi_{\text {ref }}
\end{aligned}
$$

where $\bar{N}=N_{v_{f}}+K N_{\xi}$.

\subsubsection{Integrator loop}

To track constant references for $v_{t}$ and $T_{m}$ without steady-state error, system (30) should be augmented by intermediate states $X=\left[\dot{\xi}^{T} e_{v} e_{w}\right]^{T}$ where $e_{v}=K_{v}\left(v_{t}-V_{\text {ref }}\right)$ with $K_{v} \succ 0$ as a constant gain and $e_{w}=w-w_{r e f}$ are given by

$$
\left\{\begin{array}{l}
e_{v}=g_{1}(\xi) \\
e_{p}=g_{2}(\xi)
\end{array},\right.
$$

where $g_{1}(\xi)$ and $g_{2}(\xi)$ are nonlinear functions that can be found using equations (18) and (4). In our case, these functions are linearized around an operating point to get a local approximation in the form

$$
\left\{\begin{array}{l}
e_{v}=C_{1}(\xi)+\mathcal{O}\left(\xi^{2}\right) \\
e_{T}=C_{2}(\xi)+\mathcal{O}\left(\xi^{2}\right)
\end{array} .\right.
$$

However, to inspire the standard classic IEEE governor model (7) in the closed-loop system, we modify the second integrator as follows:

$$
e_{w}=\frac{1}{T_{g}}\left(T_{r e f}-P_{s v}+K_{g}\left(\omega-\omega_{s}\right)\right)
$$

Neglecting high order terms in (42), the linear augmented system is

$$
\dot{X}=A_{a} X+B_{a} \dot{v}
$$

where

$$
A_{a}=\left[\begin{array}{cc}
A & 0_{4 \times 2} \\
C_{1} & 0_{1 \times 2} \\
C_{2} & 0_{1 \times 2}
\end{array}\right], B_{a}=\left[\begin{array}{c}
B \\
0_{2 \times 2}
\end{array}\right]
$$

The pair $\left(A_{a}, B_{a}\right)$ is proved controllable in our case where the following rank condition is verified:

$$
\operatorname{rank}\left(\left[B_{a} A_{a} B_{a} A_{a}^{2} B_{a} A_{a}^{3} B_{a} A_{a}^{4} B_{a} A_{a}^{5} B_{a}\right]\right)=6
$$


Hence, there exists a stabilizing state feedback:

$$
\dot{v}=-K_{a} X, \text { with } K_{a}=\left[\begin{array}{lll}
K & K_{v} & K_{T}
\end{array}\right]
$$

where $K \in \mathbb{R}^{2 \times 4}, K_{v} \in \mathbb{R}^{2 \times 1}$ and $K_{T} \in \mathbb{R}^{2 \times 1}$.

Integrating the above equation, the outer-loop has the following form:

$$
v(t)=-K \xi(t)-K_{v} \int_{0}^{t} e_{v}(\tau) d \tau-K_{T} \int_{0}^{t} e_{p}(\tau) d \tau
$$

It includes proportional and integral terms, which achieve zero steady-state error in power and voltage responses.

One of the interesting approaches to design the gain $K_{a}$ is the LQR (Linear Quadratic Regulator) method. The gain $K_{a}$ is computed by solving a Riccati equation and minimizes the following index

$$
J=\int_{0}^{\infty} X^{T}(\tau) Q X(\tau)+\dot{v}^{T}(\tau) R \dot{v}(\tau) d \tau
$$

where $Q$ and $R$ are the weight matrices.

\section{SIMULATION RESULTS}

In order to illustrate the performance of the proposed flatness controller in improving the transient stability of the SMIB system, some computer simulations with Matlab and Eurostag are provided here. For comparison purposes, classic controllers $[3,4]$ have also been implemented and tested, which are described by the following differential equations:

$$
\begin{aligned}
& \dot{u}_{1}=\frac{1}{T_{a}}\left(-u_{1}+K_{a}\left(v_{t}-V_{\text {ref }}\right)\right) \\
& \dot{u}_{2}=\frac{1}{T_{g}}\left(-u_{2}+K_{g}\left(\omega_{s}-\omega\right)+T_{\text {ref }}\right)
\end{aligned}
$$

To enhance the performance of the classic controller, we add a PID controller for the AVR. This PID controller will ensure an exact tracking scheme for the terminal voltage and will speed up the response time of it. Also, the classic governor controller, generating $u_{2}$, is equipped with a Fast Valving Scheme (FVS), which will enhance the transient stability [31]. Once a sever disturbance is detected, the steam valve is closed with a fast dynamic respecting the rate limitation $\left(\dot{u}_{2}=-1 / T_{c}\right)$. This situation is maintained for a time $T_{2}$ and after that the valve position is released to meet the dynamic (51) and respect the rate constraints. The parameters of the controllers are given in Table 2 .

Table 2. Parameters of the adopted controllers

\begin{tabular}{lccccccccccccc}
\hline Parameter & $\bar{u}_{1}$ & $K_{p}$ & $K_{I}$ & $K_{d}$ & $K_{a}$ & $T_{a}$ & $K_{g}$ & $T_{g}$ & $K_{v}$ & $V_{\text {ref }}$ & $w_{\text {ref }}$ & $T_{c}$ & $T_{o}$ \\
\hline Value & 3 & 10 & 1 & 0 & 1 & 0.1 & 25 & 0.3 & 5 & 1 & 1 & 0.3 & 3 \\
\hline
\end{tabular}

We consider a short-circuit in the middle of the second transmission line. Figure 2 illustrates the machine speed, mechanical torque and terminal voltage obtained from Eurostag software. The CCT in this case is $585 \mathrm{~ms}$. It is clear that the proposed approach shows appropriate robustness against hard faults occurred in the network. On the other hand, the classic controllers reveal a maximum CCT equal to $528 \mathrm{~ms}$. This means that the suggested flatness control algorithm has over $10 \%$ improvement in CCT compared to the IEEE common controllers. Also, Figure 3 to Figure 5 illustrate different state evolutions for the proposed controller against the classic IEEE controllers which have been obtained from Matlab. One can see that the proposed controller works well. In fact, the CCT obtained by the proposed controller is larger than that of the classic IEEE controllers. The applied control inputs Efd and Psv are illustrated in Figures 6 and 7, respectively. One can see that the control inputs of the proposed scheme are feasible in practice. Also, the proposed controller has the effect of fast valving as it can be observed in Figure 7, which is an interresting feature that enhances the transient stability of the power system. Finally, Figure 8 depicts the dedicated control energies for both 
the flatness and classic control strategies. It is clear that the flatness controller provides a lower value for the control energy compared to the control energy for the classic control methodology. In conclusion, the simulation results verify that the proposed flatness controller outperforms the classic IEEE controllers in both CCT and control energy criteria. It means that the flatness controller enhances the stability margin and it needs less control energy implying that its implementation costs will be much less that the existed classic ones.

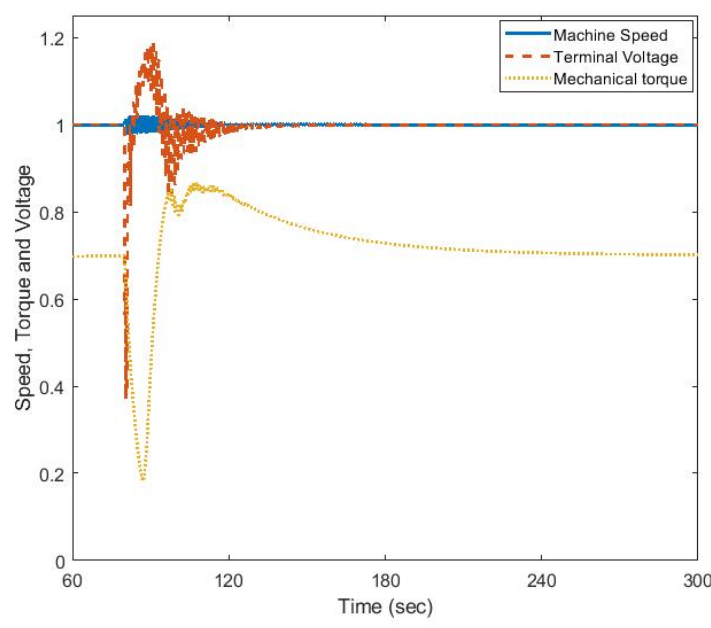

Figure 2. Speed, mechanical torque and terminal voltage response obtained in Eurostag with a $580 \mathrm{~ms}$ short - circuit at $t=80 \mathrm{~s}$

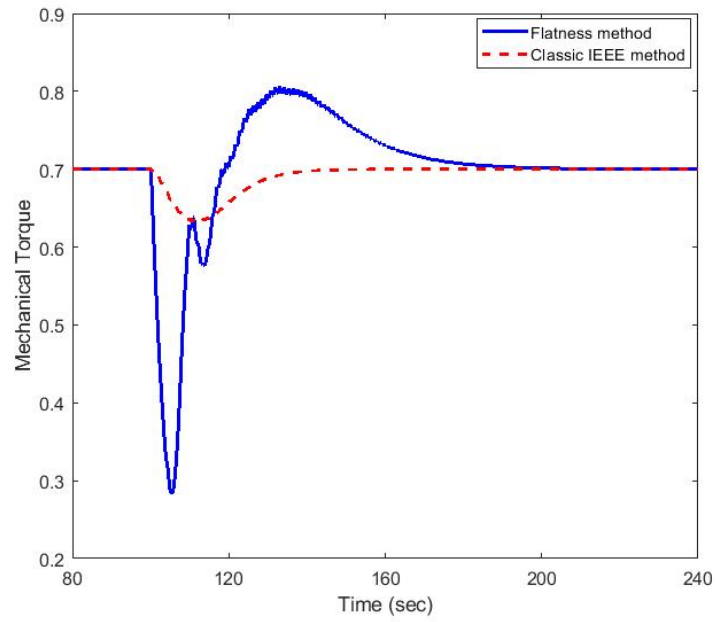

Figure 4. Mechanical torque response with a $520 \mathrm{~ms}$ short-circuit at $t=100 \mathrm{~s}$

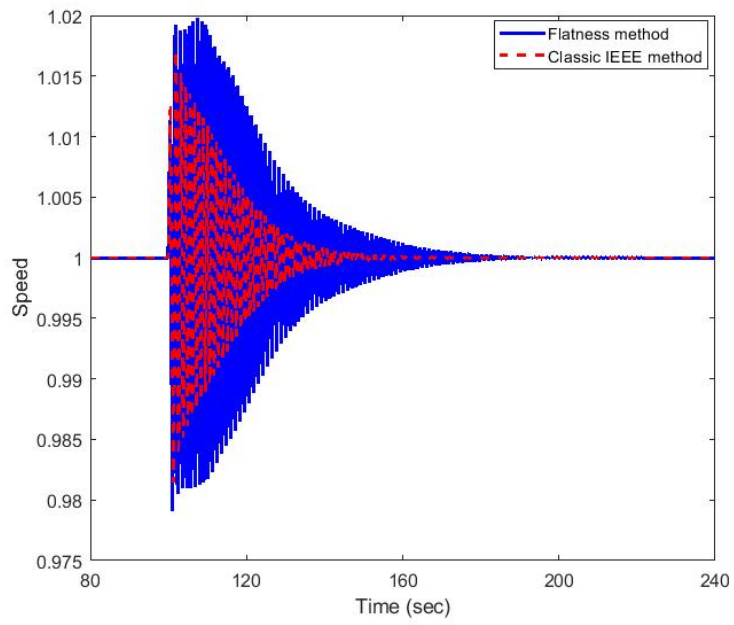

Figure 3. Speed response with a $520 \mathrm{~ms}$ short-circuit at $t=100 \mathrm{~s}$

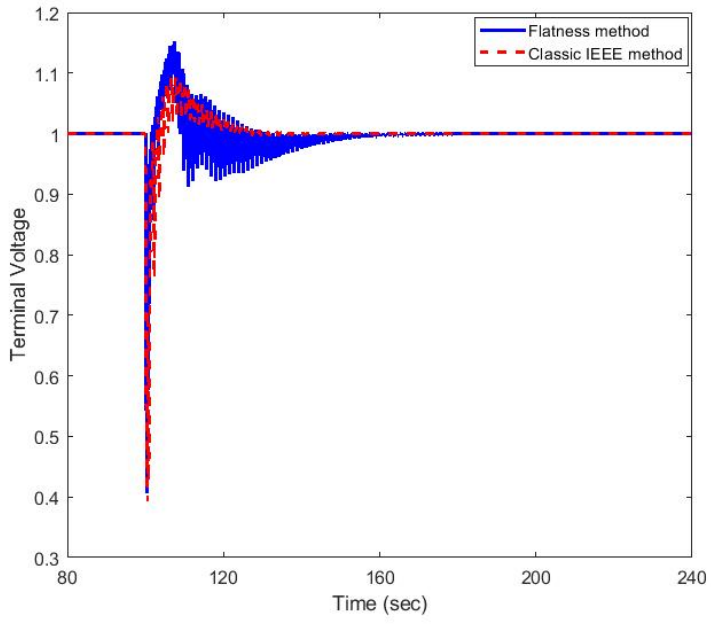

Figure 5. Terminal voltage response with a $520 \mathrm{~ms}$ short-circuit at $t=100 \mathrm{~s}$ 


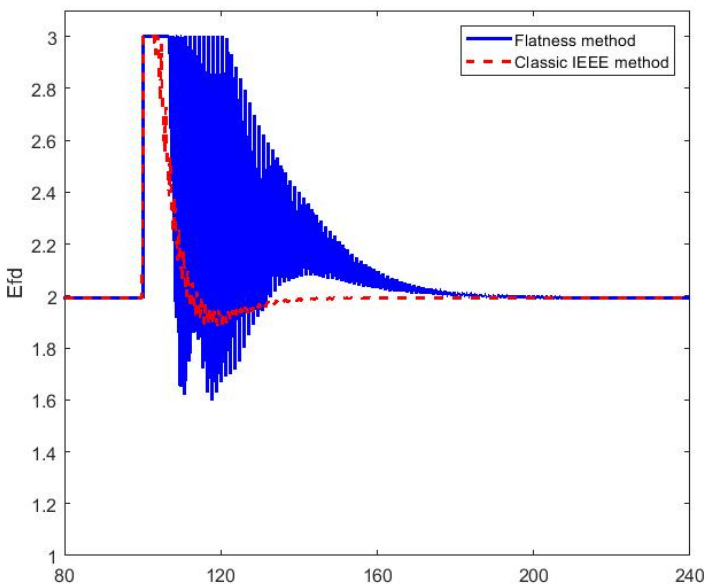

Figure 6. Efd control input response with a $520 \mathrm{~ms}$ short-circuit at $t=100 \mathrm{~s}$

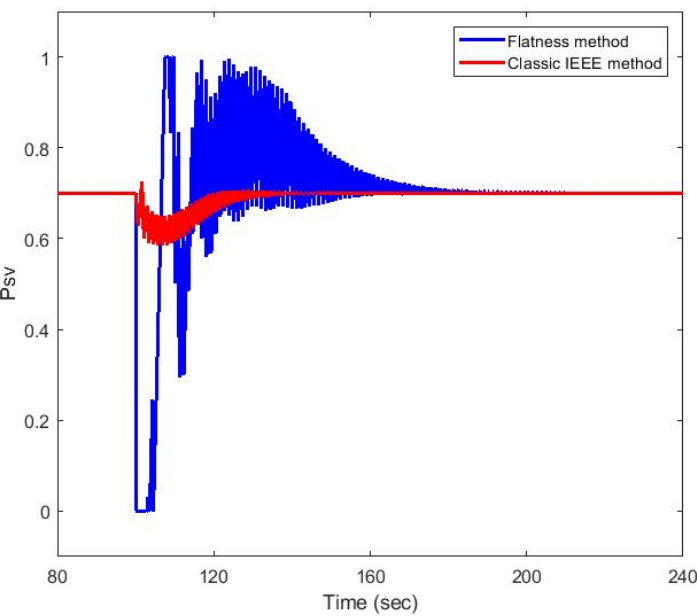

Figure 7. Psv control input response with a $520 \mathrm{~ms}$ short-circuit at $t=100 \mathrm{~s}$

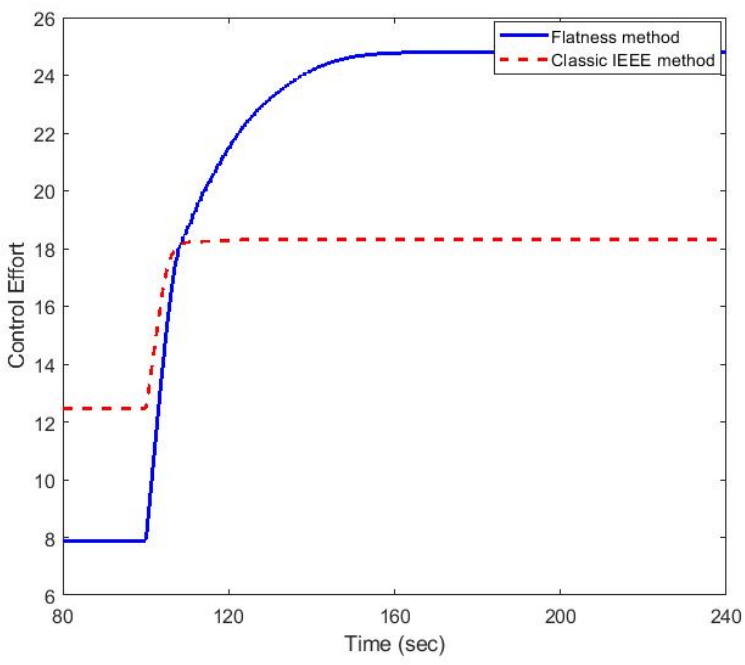

Figure 8. Control effort response with a $520 \mathrm{~ms}$ short-circuit at $t=100 \mathrm{~s}$

\section{CONCLUDING REMARKS}

In this paper, a flatness-based linearizing state feedback control scheme was proposed to enhance the stability margin of a single machine infinite bus (SMIB) system. The idea was to choose the right flat outputs to find a diffeomorphism map to make the nonlinear SMIB system equivalent to a linear canonical system. After describing the explicit differential equations governing the SMIB dynamics, the flatness controller was derived. However, since the flat controller needed all the states of the system to be available, a linear Luenberger observer was designed so that the states of the equivalent linear system were estimated. To consider the effects of the actuator saturations in the automatic voltage regulators and turbine governors, a simple method was employed to remove the nonlinearities made by these saturations. Finally, the developed observer-controller scheme was adopted to be examined under serious faults in the network. The simulation results in Matlab and Eurostag validated that the introduced methodology outperforms classic IEEE controllers in the senses of transient stability and control efforts. So, it can be concluded that the derived strategy can be utilized in real electrical networks with a satisfying robustness property. 


\section{ACKNOWLEDGEMENT}

The authors would like to thank Dr. Mohammed Benmiloud for his preliminary work on the topic during his stay at Ecole Centrale de Nantes.

\section{REFERENCES}

[1] P. W. Sauer, M. A. Pai, J. H. Chow, "Power System Dynamics and Stability: With Synchrophasor Measurement and Power," John Wiley and Sons, 2018.

[2] P. Kundur, N. J. Balu, M. G., ’Lauby, Power System Stability and Control," McGraw-hill, 1994.

[3] IEEE., "Recommended Practice for Excitation System Models for Power System Stability Studies," pp. 1-93, 2007.

[4] IEEE, "Dynamic Models for Turbine-Governors in Power System Studies," IEEE Power and Energy Society, PP. 1-117, 2013.

[5] B. R. Gamino, P. B. Araujo, "Application of a Basic Variable Neighborhood Search Algorithm in the Coordinated Tuning of PSS and POD Controllers," Journal of Control, Automation and Electrical Systems, vol. 28, pp. 470-481, 2017.

[6] R. N. D. C. Filho, V. L. Paucar, "Robust and Coordinated Tuning of PSS and FACTS-PODs of Interconnected Systems Considering Signal Transmission Delay Using Ant Lion Optimizer," Journal of Control, Automation and Electrical Systems, vol. 29, pp. 625-639, 2018.

[7] L. F. B. Martins, P. B. Araujo, E. V. Fortes, L. H., "Macedo, Design of the PI-UPFC-POD and PSS Damping Controllers Using an Artificial Bee Colony Algorithm," Journal of Control, Automation and Electrical Systems, vol. 28, pp. 762-773, 2017.

[8] Q. Su, W. Quan, G. Cai, J. Li, "Improved Adaptive Backstepping Sliding Mode Control for Generator Steam Valves of Non-linear Power Systems," IET Control Theory \& Applications, vol. 11, pp. 1414-1419, 2017.

[9] T. A. Taj, H. M. Hasanien, A. I. Alolah, S. M. Muyeen, "Transient Stability Enhancement of a Gridconnected Wind Farm Using an Adaptive Neuro-fuzzy Controlled-flywheel Energy Storage System," IET Renewable Power Generation, vol. 9, pp. 792-800, 2015.

[10] D. H. Nguyen, T. L. Nguyen, M. L. Nguyen, H. P. Nguyen, "Nonlinear Control of an Active Magnetic Bearing withOutput Constraint," International Journal of Electrical and Computer Engineering (IJECE), vol. 8, no. 5, pp. 3666-3677, 2018.

[11] A. Tripathi, K.U. Rao, L. Venkatesha, "Multi-Machine Stability Using Dynamic Inversion Technique," International Journal of Electrical and Computer Engineering (IJECE), vol. 7, no. 6, pp. 3176-3189, 2017.

[12] Y. Batmani, H. Golpîra, "Automatic Voltage Regulator Design Using a Modified Adaptive Optimal Approach," International Journal of Electrical Power \& Energy Systems, vol. 104, pp. 349-357, 2019.

[13] P. K. Ray, S. R. Paital, A. Mohanty, F. Y. S. Eddy, H. B. Gooi, ”A Robust Power System Stabilizer for Enhancement of Stability in Power System Using Adaptive Fuzzy Sliding Mode Control,'Applied Soft Computing, vol. 73, pp. 471-481, 2018.

[14] H. Yousef, H. M. Soliman, M. Albadi, "Nonlinear Power System Excitation Control Using Adaptive Wavelet Networks," Neurocomputing, vol. 203, pp. 302-311, 2017.

[15] R. Angu, R. K. Mehta, "A Single Machine Infinite Bus Power System Excitation Control Design with Extended Reduced-order Observer," Int. Journal of Dynamics and Control, vol. 6, pp. 1272-1286, 2018.

[16] H. Wang, P. He, "Transient Stability Assessment and Control System for Power System," IEEJ Transactions on Electrical and Electronic Engineering, 2019.

[17] B. C. Rout, D. K. Lal, A. K. Barisal, "Control of Period Doubling Bifurcation in an SMIB Power System Using Adaptive Controller Based on LaSalle's Invariant Principle," Cogent Engineering, vol. 4, 2017.

[18] S. Keskes, N. Bouchiba, S. Kammoun, S. Sallem, L. Chrifi-Alaoui, M. B. A. Kammoun, ”An Improved Backstepping Technique Using Sliding Mode Control for Transient Stability Enhancement and Voltage Regulation of SMIB Power System," Int. Journal of Systems Science, vol. 49, pp. 1964-1973, 2018.

[19] M. Fliess, J. Levine, P. Martin, P. Rouchon, "Flatness and Defect of Non-linear Systems: Introductory Theory and Examples," International Journal of Control, vol. 61, pp. 1327-1361, 1995.

[20] M. Fliess, J. Levine, P. Martin, P. Rouchon, "A Lie-Bäcklund Approach to Equivalence and Flatness of Nonlinear Systems," IEEE Transactions on Automatic Control, vol. 44, pp. 922-937, 1999.

[21] D.D. Silva, T. Yoneyama, "Nonlinear Control of an Aeronautical Pneumatic System," Journal of Control, 
Automation and Electrical Systems, vol. 29, pp. 292-302, 2018).

[22] S. Dominic, Y. Löhr, A. Schwung, S.X. Ding, "PLC-Based Real-Time Realization of Flatness-Based Feedforward Control for Industrial Compression Systems," IEEE Transactions on Industrial Electronics, vol. 64, pp. 1323-1331, 2017.

[23] A. Poultney, C. Kennedy, G. Clayton, H. Ashrafiuon, ”Robust Tracking Control of Quadrotors Based on Differential Flatness: Simulations and Experiments," IEEE/ASME Transactions on Mechatronics, vol. 23, pp. 1126-1137, 2018.

[24] G. Rigatos, P. Siano, P. Wira, M. Sayed-Mouchaweh, "Control of DC-DC Converter and DC Motor Dynamics Using Differential Flatness Theory," Intelligent Industrial Systems, vol. 2, pp. 371-380, 2016.

[25] M. Mehrasa, E. Pouresmaeil, S. Taheri, I. Vechiu, J.P.S. Catalão, "Novel Control Strategy for Modular Multilevel Converters Based on Differential Flatness Theory," IEEE Journal of Emerging and Selected Topics in Power Electronics, vol. 6, pp. 888-897, 2018.

[26] E. C. Anene, U. O. Aliyu, J. Levine, G. K. Venayagamoorthy, "Flatness-Based Feedback Linearization of A Synchronous Machine Model With Static Excitation And Fast Turbine Valving," IEEE Power Engineering Society General Meeting, pp. 1-6, 2007.

[27] H. A. Yousef, M. Hamdy, M. Shafiq, ’Flatness-based Adaptive Fuzzy Output Tracking Excitation Control for Power System Generators,'Journal of the Franklin Institute, vol. 350, pp. 2334-2353, 2013.

[28] M. Tacchi, B. Marinescu, M. Anghel, S. Kundu, S. Benahmed, C. Cardozo, ”Flatness-Based Feedback Linearization of A Synchronous Machine Model With Static Excitation And Fast Turbine Valving," Power Systems Computation Conference (PSCC), 2018.

[29] A. Isidori, ”Nonlinear Control Systems," Springer Science \& Business Media, 2013.

[30] E. W. Bai, K. E. Lonngren, "Synchronization of Two Lorenz Systems Using Active Control," Chaos, Solitons \& Fractals, vol. 8, no. 1, pp. 51-58, 1997.

[31] R. Patel, T.S. Bhatti, D.P. Kothari, vA Novel Control Logic for Fast Valving Operations," IEEE Power Engineering Review, vol. 22, pp. 43-46, 2002.

\section{BIOGRAPHIES OF AUTHORS}

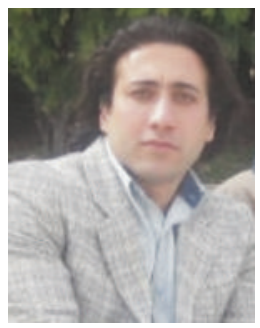

Mohammad P. Aghababa was born in Tabriz, Iran, in 1983. He received the Ph.D. degree in Electrical Engineering (major Control Engineering) from the University of Tabriz, Iran, in 2011. He is currently a Professor with the Urmia University of Technology, Iran, and a Researcher with the University of Windsor, Canada. Before joining the University of Windsor, he was a Researcher at the Ecole Centrale de Nantes, France. He has authored or co-authored over 90 international ISI-JCR journal papers. He has also authored a book entitled "Advanced Nonlinear Control Systems: Theory, Design and Applications" (in Persian). His current research interests include control theory and application, robotics and mechatronics, machine learning and AI, and fractional-order systems.

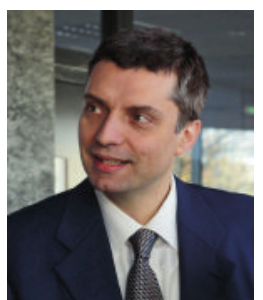

Bogdan Marinescu was born in 1969 in Bucharest, Romania. He received the Engineering degree from the Polytechnical Institute of Bucharest in 1992, the Ph.D. degree from the Universite Paris SudOrsay, France, in 1997, and the Habilitation a diriger des recherches from Ecole Normale Superieure de Cachan, France, in 2010. He is currently a full Professor and Head of the chair Analysis and control of power grids in Ecole Centrale Nantes. In the first part of his career, he was active in R\&D divisions of industry (Electricite de France and Reseau de transport d'electricte) and as a parttime professor (especially from 2006 to 2012 in Ecole Normale Superieure de Cachan). His main fields of interest are the theory and applications of linear systems, robust control and power systems engineering.

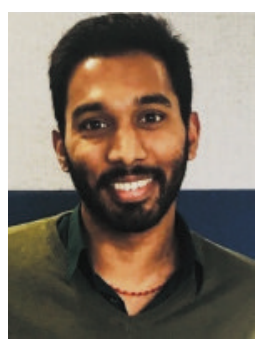

Florent Xavier Head of the Group Integration of New Technologies inside RTE's R\&D Direction, with 7 years of experience in the field of power systems. Graduated engineer from the Ecole Superieure d'Electricite; M.Sc in electrical and electronical engineering from the Swiss Federal Institute of Technology in Lausanne (EPFL) RTE representative in ENTSO-E groups: System Protection and Dynamic group (SPD), Research Development and Innovation Committee (RDIC) on System Stability working group and PG UA/MD. 